\title{
Emilia Galotti und kein Ende.
}

Von

\author{
Professor Friedrich Widder.
}

Wissenschaftliche Beilage zum Jahresberichte des Grossh. Gymnasiums in Lörrach

vom Schuljahre 1896/97.

LÖRRACH.

Progr.-Nr. 635.

Buchdruckerei von C. R. Gutsch. 1897 . 



\section{Die tragische Hamartie.}

1.

Emilia Galotti und kein Ende! Das Goethesche Wort über. Shakspeare gilt auch von Lessings Tragödie: „Es ist... schon so viel gesagt, dass es scheinen möchte, als wäre nichts zu sagen übrig, und doch ist dies die Eigenschaft des Geistes, dass er den Geist ewig anregt." Auch ,hier", sagt Erieh Schmidt $\left.{ }^{1}\right)$, ,lernt der Darsteller, der Zuschauer, der Leser nicht aus, und seit elf Jahrzehnten wogt der Streit der Meinungen um diese Tragödie, der überreich zu Theil geworden ist, was Lessing für das Grosse verlangte: zweifelnde Bewunderung, bewundernder Zweifel".

Seit nunmehr dreizehn Jahrzehnten steht denn auch das Verbaltnis Emilias zu dem Prinzen Het tore Gonzaga im Mittelpunkte des Streites, und ,sie liebt ihn, liebt ihn nicht, liebt ihn, liebt ihn nicht, sie liebt ihn" tönt uns aus allen Erörterungen abwechselnd entgegen. Es handelt sich dabei freilich um etwas Wichtigeres als um ein litterarisches Blumenorakel. Glaubten und glauben doch viele, dass geradezu die innere Wahrscheinlichkeit und zwingende Notwendigkeit der Katastrophe mit der einen oder andern Annahme steht und fallt. - Schon beim Erscheinen des Stückes hat Unzer, der Freund jenes Jakob Mauvillon, der die erste grössere Kritik über das Drama schrieb, in den gemeinsam mit diesem herausgegebenen „Briefen über den Werth einiger deutscher Dichter" (S. 49) es als einen Hauptfehler bezeichnet, dass Emilias Liebe zu dem Prinzen nicht ausgesprochen sei. „Ja! wenn uns Lessing auf eine geheime Neigung des Mädehens schliessen liesse, so wäre ihr Misstrauen gegen sich selbst

I) Lessing II, S. 1219. auch wahrscheinlicher." Goethe ${ }^{1}$ ) nannte es sodann²) gesprächsweise das ,proton pseudos" ${ }^{*}$,dass es nirgends ausgesprochen sei, dass Emilia den Prinzen liebe, sondern nur subintellegiert wird. Wenn jenes wäre, so wüsste man, warum sie der Vater umbringt. Die Liebe ist zwar angedeutet, erstlich in der Art, wie sie den Prinzen anhőrt, wie sie nachher ins Zimmer stürzt; denn wenn sie ihn nicht liebte, so hätte sie ihn ablaufen lassen; zuletzt sogar ausgesprochen, aber ungeschickt in ihrer Fureht vor des Kanzlers Hause. Denn entweder ist sie eine Gans, sich zu fürchten, oder ein Luderchen. So aber, wenn sie ihn liebe, müsse sie zuletzt sogar lieber fordern zu sterben, um jenes Haus zu vermeiden." Als die wilde, verwegene Jagd nach der tragischen "Schuld" begann, trug Goethes gewichtige Autorität das Ihrige dazu bei, dass man in einer Neigung Emilias zu dem Mörder ihres Bräntigams die tragische ćucovíce suchte und fand. Diese bis auf den heutigen Tag lebhaft verfochtene Ansicht ist auf ebenso lebhaften Widerspruch gestossen. Durch eine eingehende Würdigung und genane Abwägung des Für und Wider kam Heidemann ${ }^{3}$ ) zu dem Resultat, dass keine Stelle des Textes im ganzen Stücke direkt zur Annahme einer Liebe zwinge, dass auch ohne diese die einzelnen Scenen zu verstehen seien und der Todesentschluss Emilias verständlich bleibe. Allein mit Rücksicht darauf, dass einige Auftritte ein leichteres

1) Riemers Mitteilungen über Goethe II, S. 663 ; ich citiere nach Danzel-Guhrauer, Lessing II 2, S. 316 .

2) Goethe war also nicht, wie man vielfach liest, der erste, der auf diesen ,springenden Punkt" aufmerksam machte.

j) Über Lessings Emilia Galotti. Progr. Saarburg 1881. 
Verstāndnis zuliessen und dass der Todesentschluss eine passendere Erklärung finde, trat anch er f ür eine Neigung ein. Gleichzeitig erschien Kuno Fischers geistvolle Schrift: „Lessing als Reformator der deutschen Litteratur", worin u. a. auf Grund von Stellen, die einer Liebe geradezu widersprechen, diese mit Entschiedenheit zurückgewiesen wird ${ }^{1}$ ). Damit schien diese Frage erledigt za sein.

In neuerer Zeit haben sich nun aber wieder Stimmen für die erstere Auffassung erhoben. So Heinrich Bulthaupt in seiner „Dramaturgie der Klassiker:2) und Sigmund Schott in seinen ,Studien zur Emilia Galotti“3). Erich Sehmidt ${ }^{4}$ ) hält zwar die ganze Frage, ob Emilia den Prinzen liebe oder nicht, für schief, nimmt aber zur Erklärung der Katastrophe an, dass der Prinz in Emilia ein sinnliches, ihre Willenskraft lahmendes Wohlgefallen erweckte. Man legt dabei besonderen Wert auf jene erregte Erzăhlung Emilias von dem Vorfall in der Kirche (II, 6), in der schon M. Bernays ${ }^{5}$ ) einen Verrat ihrer Leidenschaft und einen Kampf ihres Herzens mit einer Liebe zn dem Prinzen hat entdecken wollen.

Emilia: . . Und da ich umwandte, da ich i h n erblickte -

Claulia: Wen, meine Tochter?

Emilia: Rathen Sie, meine Mutter, rathen Sie. - Ich glaubte, in die Erde zu sinken. - I h n selbst.

Schon jenes ,ih n* halten die einen, und namentlich dieses "Inn selbst" die andern für bezeichnend, für ein Zeichen nåmlich dafür, dass Emilias ganzes Sein und Denken so von der einen Gestalt erfüllt ist, dass sie unbewusst dieselbe Voraussetzung bei der Mutter macht. Nun ist doch auf den ersten Blick klar, dass zu jenem ,i ihn", dem die Worte vorausgehen: ,Ich zitterte, mich

I) I, S. 250 ff. und S. 209.

2) 12, S. $25 \mathrm{ff}$.

3) Beilage zur Allg. Zeitung 1890 Nr. 42 und 43 .

4) II, 1, S. 200 und 201 und II, 2, S. 800.

5) Morgenblatt 1863 Nr. 13 und 14. Vergl, auch Heidemann S. 12. umzukehren, ich zitterte, ihn zu erblicken, der sich den Frevel erlauben dürfen", dieser letzte Relativsatz zu ergänzen ist, dass nicht betont werden kann: ,da ich thn erblickte", sondern: „da ich ihn erblickte" als Gegensatz zu: ,ich zitterte, ihn zu erblicken", wie ja auch: ,da ich mich umwandte" dem, ich zitterte mich umzukehren" gegenübersteht. Aber dieses ,verräterische" ,Ihn selbst"! ,Der Prinz", sagt Erich Schmidt, ,der heute zum ersten Mal seine Liebe entdeckt, muss doch Emilias Gedanken sehr beschäftigen, um für sie ,er selbst" zu sein." Und Bulthaupt: ,Der Geliebte mag ,er selbst" sein ; aber der Prinz ron Guastalla, der dieses Mädchen, den dieses Mädchen ein einziges Mal in jener Vegghia bei dem Kanzler Grimaldi gesehen hat? Würde Graf Appiani für Emilia Galot'ti „er selbst" gewesen sein ?." Sagte Emilia an di e se r Stelle bloss „I h n", so möchte darunter ganz wohl eben i h r ,Er", der Gebieter ihres Herzens, gemeint sein können, und Erich Schmidts Hinweis auf Nathan $I, 4^{1}$ ) bestünde zu Recht. So aber heisst es "Ihn selbst", und da liegt doch wohl die Auffassung viel näher, dass kein geringerer als Er, der Prinz, der Gebieter des Landes, nicht ihres Herzens, der Frevler ist. Just dieses , selbst" wird auch an anderen Stelien des Stückes zur Hervorhebung der Person des $\mathrm{Pr}$ inzen angewendet:

"Ich will wetten, dass der Prinz schon selbst um lhre themre, ehrwürdige Mutter ist" sagt Marinelli zu Emilia (III, 4), und zu Claudia (III, 8) :

,Mit der zärtlichsten Sorgfalt ist der Prin z selbst um sie beschäftigt" -

„Wer? - Wer selbst ?.

Dieses ,s e lb st" ist an unserer Stelle um so ange-

1) Daja: Er lïsst sich wieder sehn! Er lässt Sich wieder sehn!

$$
\begin{aligned}
& \text { Nathan: } \quad \text { Wer, Daja? wer? } \\
& \text { Daja: } \\
& \begin{array}{l}
\text { Nathan: } \\
\text { Er? er? - Wann lässt sich der nicht sehn - Jaso, } \\
\text { Nur euer Er heisst er. }
\end{array}
\end{aligned}
$$


brachter, als es das Schlussglied einer ganz natürlichen Steigerung ist. Erst ist es ein unbestimmtes, ,e twa s", das dicht hinter Emilia seinen Platz nahm; es sprach ron Schönheit, von Liebe, es klagte, es beschwor. Nach diesen Liebesbeteuerungen wird es dann $\mathrm{zu}$ einem bestimmteren e $r$, den sie $z$ erblicken zitterte. Und endlich, da sie sich umwandte, da sie ihn erblickte, ist es - ,er selb st". - Indes, es führt noch eine andere Erwägung zur Haltlosigkeit jener Vermutung. Das ,I $\mathrm{hn}$ selbst* ist die Antwort auf die Frage der Mutter. Es handelt sich also nicht darum, wer ,für E m i l i a", sondern wer für die Mutter, ,er selbst* ist. Die Worte: „R a the n Sie, meine Mutter, rathen Sie: lassen vollends keinen Zweifel darüber, dass mit "Ihn selbst" auf eine Person hingedentet ist, die herauszufinden für die Mutter keine besondere Schwierigkeit sein muss. Was ist denn natürlicher, als dass die ,eitle, thörichte Mutter" jene unter ihren Augen der Tochter dargebrachten Huldigungen des Prinzen, von denen sie dem strengen Odoardo ,in einem Tone der Entzückung“ erzählt (II, 4), wieder und wieder zum Gegenstand des Gespräches mit Emilia gemacht hat? Ja, sie hat so oft davon gesprochen, dass sie nicht eimmal mehr weiss, ob sie es Odoardo schon mitteilte. „Denn $\mathrm{h} \mathrm{ab}$ i ch Dir sehon gesagt, dass der Prinz unsere Tochter gesehen hat?:* (II. 4). "Wen" also ,ihn selbst?.. Nun, wenn denn anders als: eben den (Prinzen), von dem Sie mir so oft gesprochen, der, wie Sie wissen, sich mir gegenüber ,so gnädig bezeigte ". Übrigens ist diese Beziehung dem - um mit Goethe zu sprechen - „Luder* von selbstgefällig ausfragender Mutter wohl ebenso klar, als sie es dem Leser oder Zuschauer sein muss, der fast unmittelbar vorher die Mitteilungen Claudias an Odoardo mit angehört hat.

Einen weitern Beweis für die Neigung Emilias zum Prinzen findet man in dem Verhäl $\mathrm{n}$ is z w is chen A p piani und Emilia. Es herrseht, heisst es, zwischen den beiden Verlobten ein Ton ehrerbietiger Reserve, aber niemals flammt Herz in Herz. Ein sonderbarer Hochzeiter, dieser Appiani, kein feuriger Liebhaber. Warm und feurig wird er - nicht von den Reizen seiner Braut hingerissen, sondern sobald das Gespräch auf ihren Vater kommt. Ja, nicht einmal eine ordentliche Liebeserklärung bringt er fertig. Und Emilia? Nun, auch sie spricht ja nur ron ,,dem Grafen", von dem ,guten Appiani", nennt ihn „Herr Graf", niemals „Bräutigam" oder - ,Gatte". Also zu wenig Fener und Flamme, zu wenig schwärmerische und schwungvolle Liebesbeteuerungen! Als ob alle Liebespaare Romeos und Julien sein müssten und - was die Hauptsache ist - als ob es nicht im $\mathrm{Charakter}$ Appianis und Emilias begründet wäre, dass sie es nicht sind! Appiani, der „ernste, würdige", bescheidene, zurückhaltende Mann ist allerdings kein feuriger Liebhaber; auch haben ihn andere Vorzüge, nicht wie den Prinzen die „Reize" zu Emilia hingezogen. „So recht, meine Emilia! Ich werde eine fromme Frau an Ihnen haben, und die nicht stolz auf ihre Frömmigkeit ist* (II, 7). Warum sollen wir es denn da Claudia nicht glauben, dass die Liebe zusammenbrachte, ,was für einander geschaffen war", und wenn wires dieser nicht glauben wollen, warum nicht Odoardo, dass sie sich gefunden haben, ,die f'ür ein an der bestimmt waren"? (II, 4). Aber weil der ,feurige": Prinz nun einmal durchaus Eindruck gemacht haben muss, deswegen darf der ernste Appiani keinen gemacht haben. Wenn dann Emilia diesen „Herr Graf" anredet, von ihm als dem ,Grafen" spricht, so ist das ebenso wenig auffallend, als wenn Odoardo und Claudia sieh gegenseitig und Emilia ihre Eltern mit „Sie" anreden. Überdies sagt sie ja doch auch ,mein lieber Graf.", mein ,guter Appiani", wie er "meine Emilia“. „Man prüfe," sagt Schott, ,die einzige Scene, in welcher Emilia und ihr Verlobter zusammen sind, auf ihre gegenseitigen Empfindungen, und man frage sich, ob dieser die Haltung eines Bräntigams am Hochzeitsmorgen einnimmt." Thun wir das, anstatt fortwährend mit dem Feuer zu spielen, und lassen wir gerade den Schluss dieser Scene (II, 8) unbefangen auf uns einwirken: 
Emilia; Und was meinen Sie, das ich mir ausgedacht habe? - Was trug ich, wie sah ich aus, als ich Thnen zuerst gefiel? - Wissen Sie es noch?

Appiani: Ob ich es noch weiss? Ich sehe Sie in Gedanken nie anders als so, und sehe Sie so, auch wenn ich Sie nicht so sehe.

Emilia: Also ein Kleid von der nămlichen Farbe, von dem nämlichen Schnitte, fliegend und frei -

Appiani: Vortrefflich!

Emilia: Und das Haar -

Appiani: In seinem eigenen braunen Glanze; in Locken, wie sie die Natur schlug -

Emilia: Die Rose darin nicht zu vergessen!Recht! - recht! - Fine kleine Geduld, und ich stehe so ror Ihnen da!

Da sprüht allerdings kein Feuer, da flammt nicht Herz in Herz. Aber wir stellen die Gegenfrage: Ist das wirklich nur der Ton ehrerbietiger Reserve und nicht vielmehr der stillseligen, herzinniglichen Liebesglüeks, ein dem Charakter dieses Liebespaares völlig entsprechender Ton, der Dutzende der feurigsten Liebesschwüre anfwiegt?

Man nimmt ferner Anstoss an dem Verhalten Emilias nach Appianis Tode und sagt: „Wem diese Verbindung Emilias ganzes Glück ausmachte, wenn dieser Mann ihr ganzes Herz ansgefüllt haben würde, so komnte sie nach seinem Tode so ruhig nicht sein, wie sie es in Wirklichkeit ist. Denn als sie es erfahrt, dass der Graf tot ist, kein Wort der Klage, keine Thräne: nur unmittelbar die Frage: „.Und warum er tot ist? Warum ?*: Eine wirklich liebende Braut hatte doch ihrem Schmerze Ton verliehen, ehe sie an die Verstandesfrage "Warum ?". gedacht hätte". Das eine Mal zu wenig Feuer und Flamme, das andere Mal zu wenig Trauer und Thränen! Mag sein, dass ,eine* andere Braut in ihrem Schmerze zusammengebrochen wäre; aber das ist nun einmal nicht $\mathrm{Em}$ il i a s "A rt" (IV, 8); denn, sagt die Mutter, ,sie ist die Furchtsamste und Entschlossenste unseres Geschlechts. Threr ersten Eindrücke nie mächtig, aber nach der geringsten Utberlegung in alles sich findend, auf a lle s gefasst." Haben wir es aber hier $(V, 7)$ mit „e r st e n Eindrücken" zu thun? Hat sie denn nicht schon mit dem Gedanken sich vertraut gemacht, dass ,alles verloren" ist, dass sie ,wrohl ruhig sein muss"? Hat sie denn nicht schon , die ganze schreckliche Geschichte in den nassen Angen ihrer Mutter gelesen"? Ob diese Ruhe mit dem EwigWeiblichen vereinbar ist oder nicht, darüber mögen die Kritiker streiten, mit wem sie wollen. Unbestreitbar ist und für uns die Hauptsache, dass diese Ruhe ihrem Charakter entspricht, eben ihre "A r t" ist. "Die Gesinnungen". heisst es in der Hamburgischen Dramaturgie (II), ,müssen in dem Drama dem angenommenen Charakter der Person, welche sie ãussert, entsprechen; sie können also das Siegel der a b s olut e n Wahrheit nicht haben; genug, wenn sie poetisch wahr sind." - Aufiallend soll auch sein, dass Emilia in der grossen Bestürzung mehr von ihrer Mutter als von ihrem Verlobten spricht, dass sie nach der Ermordung Appianis, als Emilia ausser Atem ins Schloss geführt wird, znerst. fragt : "Wo bleibt meine Mutter" und dann erst: „Wo bleibt der Graf?.. Das alles soll beweisen, dass nicht die allbenvingende Liebe Emilia mit Appiani verbunden gehabt. Nun könnte es an sich noch lange keine Gleichgiltigkeit gegen ihren Bräutigam bedenten, wenn eine Braut in solcher Lage zuerst an ihre Mutter dãchte. Aber man sehe sich doch die Stelle (II, 4) einmal unbefangen an: ,Aber Gott, Gott! wo bin ich? - Und so ganz allein? Wo bleibt meine Mutter? Wo bleibt der Graf? Sie kommen doch nach? mir auf dem Fusse nach ?:•

Aus diesen erregten, hastig hervorgestossenen Fragen, in denen sie die ihrem Herzen gleich nahestehenden Personen so zu sagen in einem Atem nennt, eine Gefühlslokation herauslesen wollen, das heisst denn doch den, rechnenden" Dichter überrechnen. Ausserdem stimmt diese Lokationsberechnung nicht einmal. Denn gleich nachher, wo der Schuss erwähnt wird, sagt Emilia: "Und das hat den Grafen oder meine M u t t e r getroffen." Allein darin soll gerade 
der Witz liegen. Diese einzige Umstellung scheint Schott eine „psychologische Feinheit des denkenden Dichters." Denn bei dem Gedanken an ein Unglück zagt und scheut sie, den Namen der ihrem Herzen näher stehenden Person zu nennen. Schott selbst scheint bei dieser Interpretationsweise etwas bange geworden zu sein. Ist er doch darauf gefasst, dass man seinen Versuch mit der Bezeichnung „Deuterkunststïeke" abzufertigen suchen wird. Für die pedantische Art, mit der hier in die lebendig bewegte Rede peinlich nüchterne Erwägungen hineingeheimnisst werden, ist das eine verhältnismässig noch recht wohlklingende Bezeichnung.

Es wird also schon dabei bleiben müssen, dass keine Textesstelle zur Annahme einer Liebe Emilias zum Prinzen zwingt. dass aus den angeführten Stellen nicht einmal dann eine solche Neigung herausgelesen zu werden brauchte, wenn diese aus andern Gründen unzweifelhaft feststünde. Für den letztern Fall nun ruft man die Schauspielkunst zu Hilfe. Sie soll dem Verständnis des Zuschauers nahe legen, was des Dichters Worte dem Leser und Hörer vorenthalten haben. Heidemann weist dabei hin auf E. Deurients Worte ${ }^{1}$ ): „Dies Stück vollendete die Wohlthaten, welche Lessing der Schanspielkunst erwiesen. Er gab ihr darin Charaktere, welche an innerm Reichthum und Vollendung von keinem spätern Dichter übertroffen worden sind und dennoch den Darsteller so viel zwischen den Zeilen zu lesen, zu errathen und zu ergänzen übrig lassen. An sämtlichen Rollen von Emilia Galotti kommt die Schanspielkunst niemals zi Ende, sie findet unerschöpfliche Anregungen und Aufgaben darin." Man citiert ferner den Ausspruch Goethes: „Wenn alle so vortrefflich spielten, wie Madame Wolf .... dass sie diese Maske ausfülten, ja noch mehr dahinter zu errathen liessen, so würde man nicht wissen, was man zu sehen bekäme, so gewönne alles mehr Sinnlichkeit" u. s. w. Was sagt denn aber Lessing selbst dazu? In einer Note zu demselben Brię, in dem er wünscht, dass

1) Geschichte der deutschen Schauspielkunst II, 251 .
Lessing von der Verführung etwas auf dem Theater hătte vorgehen lassen sollen, erzählt Nicolai ${ }^{1}$ ): „Über eine andere Anmerkung, die ich Lessingen damals - bei seiner Anwesenheit in Berlin 1775 mittheilte, wäre er beinahe böse geworden. Madame Starkin, die grosse Schauspielerin, hatte mir einmal gesagt: , „die Rolle der Emilia könne nie gespielt werden, so wie sie gespielt werden sollte; demn sie erfordere ein ganz junges Mädchen, die doch die vollkommenste Schauspielerin sein müsste, um der Rolle Genüge zu thun" ". Diese Bemerkung theilte ich Lessingen mit und setzte hinzu: es möchte diese grosse Schauspielerin wohl Recht haben. Lessing rief aus: ,,, Hol der $\mathrm{T}-$ - die Frau mit ihrer Bemerkung! Die Rolle der Emilia erfordert gar keine Kunst! Naiv und natürlich spielen kann ein junges Mädchen ohne alle Anweisung":".

Nach all' dem dürfte in dieser Frage nicht ohne Interesse sein ein Vergleich mit Lessings Virginia und der des spanischen Dichters Montiano y Luyando, einer Tragödie, die namentlich in der Gestaltung der Charaktere Odoardos und Emilias von nachhaltigem Einfluss auf Lessing war²). In jener sagt Clandius zu Rufus ${ }^{3}$ ): ,Du weisst, sie ist versprochen, mit dem jungen Icilius versprochen, und wie zärtlich liebt sie ihn, dieses Schosskind des Volkes ... ." Und in dem Auszug, den Lessing in seiner Theatralisehen Bibliothek von der Dichtung des Spaniers giebt, heisst $e^{\dagger}$ ): ,Nicht zwar, als ob sie (Virginia) sich fürchte, sich von dem Appius erweichen zu lassen, nein, ihr Herz ist einzig und allein mit dem, was sie dem Icilius, dem sie von ihrem Vater versprochen worden, schuldig ist, erfüllet und gänzlich unfühig, irgend einen andern Eindruck aufzunehmen .... Sie beklagt ihr Schicksal, welches sie ihrem Vater-

1) Hempel XX, 2, 586. Danzel-Guhrauer II $2,325$.

2) Volkmann ,Zu den Quellen der Emilia Galotti" in der Festschrift zur 50jährigen Gedenkfeier etc. des Realgymnasiums in Düsseldorf 1888.

s) Hempel IX, 2 S. 632.

4) Hempel XI, 1 S. 256, 258. 
lande zu einem traurigen Schauspiel mache, ohne dass sie sich gleichwohl das Geringste in ihrer Liebe für den Icilius in ihren Gedanken und Handlungen vorzuwerfen habe." Also: sie liebt ihn nicht! 2.

Wenn nun aber nur mit der Annahme einer Liebe alles ,planmässig, klar motiviert und forgerichtig" sein soll, verlieren wir denn da trotz alledem mit ihrer Verwerfung nicht zu viel? Man bedenke: „Welch' ein tragischer Conflict, wenn die Unglückliche sich gestehen muss, dass die Kugel des Bravo im Grunde ihr eigenes Herz nicht mitverwundet hat, da es dem Todten nur aus Pflicht sich zugeneigt hatte, dass durch dieses Verbrechen ein Weg frei gemacht wird, der zu einem heimlich ersehnten Ziele führen würde und den nur ihre Ehre zu betreten ihr verbiete! ${ }^{\prime \prime 1}$ ) Welche tragische Schuld, wenn Emilia das Bewusstsein hat, „im eigenen, tiefsten Herzen dem Prinzen gegenüber nicht ganz frei zu sein ${ }^{* 2}$ ), wenn, ihr Gewissen ihr Vorwürfe macht, dass sie, die Braut eines so trefflichen Mannes, wie Appiani geschildert wird, einem Gefühle für den Prinzen Raum gab!:3). Und eine tragische Schuld muss doch Lessing angenommen haben, wemn anders sein auf Aristoteles abgelegtes Glaubensbekenntnis ernst gemeint und sein Drama die Probe auf das in der Hamburgisehen Dramaturgie aufgestellte Exempel sein soll. Jndes ist auch von dieser Seite her die Annahme einer Liebe zum allermindesten nicht notwendig. Nur muss man die aristotelische áucoria nicht einseitig als „moralische" Schuld auffassen, die man mit Hilfe des Strafgesetzbuches oder der bürgerlichen Moral beurteilt, muss auf den Glauben an das Dogma von der poetischen Gerechtigkeit verzichten wollen, der in jeder Tragödie eine ,adaequate Schuld", eine gerechte Verhaltnismässigkeit von Schuld und Sühne findett). Vor allem aber muss betont werden, dass Lessing selbst die Hamartie in diesem Sinne nicht fasste. Am

1) Paul Heyse in einem Briefe an Schott. Allg. Zeitung a, 0. 0.

2) Heidemann, S. 12

5) Nolting, Über Lessings E. G. Progr. Wismar, 1878 S. 5.

4) Wie z. B. Günther, Gruadzüge der tragisehen Kunst. klarsten geht dies aus dem Briefe an Moses Mendelsohn vom 18. Dezember 1756 hervor $\left.^{1}\right)$ : „Unterdessen ist es doch auch wahr, dass an dem Helden eine gewisse $\alpha \mu \alpha \varrho x i \alpha$, ein gewisser Fehler sein muss, durch welchen er sein Unglück über sich gebracht hat. Aber warum diese áucostia, wie sie Aristoteles nennt? Etwa, weil er ohne sie vollkommen sein würde und das Unglück eines vollkommenen Menschen Abscheu erweckt? Gewiss nicht. Ich glaube, die einzige Ursache gefunden zu haben, sie ist diese: weil ohne den Fehler, der das Unglück über ihn zieht, sein Charakter und sein Unglück kein Ganzes ausmachen würden, weil das eine nicht in dem andern gegründet wäre und wir jedes von diesen zwei Stücken besonders denken würden." Und aus der Hamburgisehen Dramaturgie (82) hoben B. Arnold ${ }^{2}$ ) und K. Fischer ${ }^{3}$ ) den Satz hervor: „Ein Mensch kann sehr gut sein und mehr als eine Schwachheit, mehr als einen Fehler begehen, wodurch er sich in unabsehliches Unglück stūrzt, das uns mit Mitleid und Wehmuth erfüllt, ohne im geringsten grässlich zu sein, weil es die natürliche Folge seines Fehlers ist" ". Diesen ,Fehler" sehen beide mit Recht darin, dass Emilia aus Gehors a m gegen ihre Mutter das Zusammentreffen mit dem Prinzen und das Erlebnis in der Kirche ihrem Bräntigam in der besten Absicht verheimlichte. Denn hätte Appiani nur eine Andeutung empfangen, so konnte der Mord nicht geschehen, die Unterlassung Emilias ,verschuldet" den Tod Appianis. Dem gegenüber verhallt wirkungslos des Grafen $v$ ग Schack kraftgenialisches Donnerwort ${ }^{4}$ ): „Man muss ein ron åsthetischer Duselei ganz erweichtes Gehirn haben, um nicht einzusehen, dass eine Schuld, die einen tragischen Untergang herbeiführen soll, auch eine entsprechend schwere sein müsste, wie die ron Macbeth oder Wallenstein, und dass irgend eine leichte Verschuldigung, wie man sie etwa für

1) Hempel XX, 1 S. 90.

2) Lessings E. G. in ihrem Verhältnis zur Poetik des Aristoteles und zur Hamb. Dramat. S. 15.

3) S. 198.

4) Perspektiven I, S. 4. 
Emilia Galotti austüfteln konnte, hiezu nicht ausreicht." Aber auch Heidemanns und Schotts Bedenken fallen, dass derselbe Lessing Geh or s a m als Schuld soll angesehen haben, der über eben diese Emilia Galotti an seinen Bruder Karl schreibt ${ }^{1}$ ): ,Ieh kenne an einem unverheiratheten Mädehen keine höheren Tugenden als F r ö m migke it und $\mathrm{G}$ e h o r s a m."Denn dass auch Tugenden, iu reinstem und edelstem Wollen ausgeführte oder unterlassene Handlungen unendliches Weh herbeifuhren, dass die Personen der Tragỏdie ihr Leid ,,verschulden“, wenn auch nicht verdienen, dieses Schuldlos-schuldig, das ist eben der Fall der Tragödie. Wenn Deianira, um den von ihr mit aller Hingebung und Treue geliebten Herakles noch fester an sich zu ketten, diesem das Nessosgewand überschickt und ihm dad u re h den qualvollsten Tod bereitet; wenn Kriemhilde, um ihren Siegfried vor jedem Unfall zu bewahren, ihm in zärtlich liebender Sorgfalt das Kreuzchen auf das Gewand năht und da d u reh seinen Tod verschuldet ${ }^{2}$ ): so liegt darin, in diesem Gegensatz von guter Absicht und schlimmen Folgen, erschütternde und ergreifende Tragik und eine der vornehmsten Quellen tragischen Mitleids.

Möglich, sicher vielleicht, dass sich jene Unterlassung Emilien später als Schuldbewusstsein aufdrängt. Dass sie dieses aber auch ausspricht, ,in schonungsloser Selbstanklage sich leidenschaftlich vorrüekt", wenn sie in der vorletzten Scene auf die Frage des Vaters: „Was nennst Du alles verloren? Dass der Graf todt ist?:. antwortet: ,Und warum er todt ist! W a r u m !* vermögen wir Kuno Fischer ${ }^{3}$ ) und Erich Schmidt ${ }^{4}$ ) u. a. nicht zuzugeben. Dies ,W a r $\mathrm{um}$ " kann nicht den Sinn haben: weil ich Appiani das Zusammentreffen mit dem Prinzen verheimlichte, schon deswegen nicht,

'1) 10. Februar 1772 Hempel XX, I S. 48?.

2) Mit klassischer Schärfe und Kärze bezeichnen diesen tragischen Kontrast die Worte des Nibelungenliedes:

si wânden helt vristen: ez was ùf sinen tôt getân.

s) ล. o. 0 .

4) II, 1 S. 203 น. 213. weil gleich nachher Emilia an diese Worte die Aufforderung zu schleuniger Flucht knüpft :

„Denn, wenn der Graf todt ist, wenn er d a r u m todt ist - dar um! was verweilen wir noch hier? La s sen Sie un flie he n, mein Vater!.

Dieses „Warum", dieses ,Darum“, dieses Etwas, was sie nicht auszusprechen wagt, kann somit nur bedeuten: weil der Prinz, in dessen Schloss wir uns befinden, durch die Ermordung des Grafen die Bahn hat frei machen wollen für sein frevles Begehren. Nicht also eine Selbstanklage liegt in jenem Ausrufe, sondern ein Hinweis auf die ungeheure Gefahr, der sie entfliehen müssen.

Noch eine weitere Folgerung, die Kuno Fischer an diese Hamartie Emilias knüpft, scheint uns nicht gerechtfertigt zu sein: "Wie kann," sagt $\mathrm{er}^{1}$ ), „Appiani auf die fürstlichen Wünsehe, die der Höfling ihm überbracht hat, überhaupt eingehen?:*

„,.Die Gnade des Prinzen, die Ihnen angetragene Ehre bleiben, was sie sind; und ich zweifle nicht, Sie werden sie mit Begierde ergreifen." *

",A 11 erding s"..,

antwortet der Graf, ,, nach einiger Überle gung ${ }^{* *}$ (II. 10). lch möchte wissen, w a s er sich überlegt hat? Gesagt wird es nicht; es ist schwer zu errathen. Aus dem Charakter und der Sinnesart Appianis wäre zu vermuthen, dass er den Antrag einfach ablehnte. Auch sehe ich nicht, wie er beim besten Willen denselben ausführen wird, da er schon in der nächsten Stunde heirathen und sogleich auf seine văterlichen Güter nach Piemont abreisen will. Fassen wir ihn und seine Situation a 11 e in ins Auge, so ist jenes überlegte ..Allerdings" eine so befremdliche Wendung, dass man versucht sein könnte, sie aus einem anderweitigen (weniger Appiani, als vielmehr) dem Dichter unentbehrlichen Motiv zu erklären. Hätte der Graf gewusst, was ihm Emilia verschwiegen, so würde er den Antrag Marinellis auf eine Art zurückgewiesen haben, die dem letztern sein Concept vollig verrücken musste. Der Dichter lässt ihn daher einen Sehritt

1) 11 S. 230 und 231 . 
thun, der unmóglich war, wenn er die Absichten des Prinzen gekannt hätte: einen Schritt, der als die nächste Folge der Unterlassung Emilias erscheinen soll und ers che int. Dass Appiani diesen unwillkommensten und ungelegensten aller Aufträge erfüllen will, um dem Prinzen einen Dienst zu erweisen, zeigt uns den völlig arglosen Mann, der dem Spiele Marinellis verfällt, während ein Wort seiner Braut ihn davor bewahrt hätte. Die Schuld Emilias wird dureh Appianis arglose Bereitwilligkeit sofort erleuchtet: dies war die A bsicht des Dichters, aber das Motiv Appianis bleibt fraglich." Fraglos ist, dass Appiani seinem $\mathrm{C}$ h a ra $\mathrm{k}$ ter gemåss auf den Antrag nicht eingehen kann. Wie, wenn er es mit der Antwort "Allerdings" auch gar nicht thăte, wenn dieses ,Allerdings" eine im Ernste zustimmende Antwort auch gar nicht enthielte? Der Mann, der zu dem Höfling das stolze Wort spricht: ,Ein Herr, den man sich selber wählt, ist unser Herr so eigentlich nicht. Ich gebe zu, dass S i e dem Prinzen unbedingtern Gehorsam schuldig wären. Aber nicht i $\mathrm{ch}$. Ich kam an seinen Hof als Freiwilliger. Ich wollte die Ehre haben, ihm zu dienen, aber nicht sein Sklave werden. Jch bin der Vasall eines grössern Herrn", der kann Redensarten wie: „Die Gnade des Prinzen, die Ihnen angetragene Ehre bleiben, was sie sind, und i $\mathrm{h}$ $\mathrm{z}$ weifle $\mathrm{nicht}$, Sie werden sie mit Begie rde ergreifen" nur mit einem ironisch zustimmenden, mit einem die Gesinnung Marinellis und dessen Worte ironisierenden, Allerdings" beantworten. Was hätte er auch antworten sollen? Im Ernste zustimmen kamn er nicht, schlechthin ablehnen will er nicht, bevor die "plaudernde Hofschranze* sich ausgeplaudert hat. Darum giebt er - und das ist das Resultat seiner kurzen,,$\breve{\mathrm{C}}$ b erleg ung* eine doppelsinnige Antwort, die Marinelli vorerst als Zustimmung auffassen mag. Dieser die Denkungsart Marinellis ironisierende Ton kehrt ja anch gleich nachher wieder:

Appiani: Und ich hoffe, dass der Prinz selbst meine Entschuldigung wird gelten lassen.

Marinelli: Die bin ich begierig zu hören.

Appiani: 0, eine Kleinigkeit. Sehen Sie, ich soll noch heut eine Frau nehmen.

Die Ansicht aber, dass jene verhängnisvolle Unterlassung Emilias tragische "Schuld" ist. beibt. nichtsdestoweniger unangetastet bestehen.

\section{Die tragische Katastrophe.}

1.

Mit der Annahme einer Liebe oder sinnlichen Bezauberung glaubte man endlich auch den Schlüssel gefunden zu haben zu einer passenden Erklärung jener vielumstrittenen Worte Emilias, mit denen sie Odaardos Vertrauen anf ihre Entschlossenheit und Unschuld, ,die über alle Gewalt erhaben ist", verwirft: „Aber nicht über alle Verführung. Gewalt! Gewalt! Wer kann der Gewalt nicht trotzen? Was Gewait heisst, ist nichts: Verführung ist die wahre Gewalt. Ieh habe Blut, mein Vater; so jugendliches, so warmes Blut, als eine. Auch meine Sinne sind Sinne. Ich stehe für nichts. Ich bin für nichts gut. Ich kenne das Haus des Grimaldi. Es ist das Haus der Frende. Eine Stunde da, unter den Augen meiner Mutter; - und es erhob sich so mancher Tumult in meiner Seele, den die strengsten Übungen der Religion kaum in Woehen besänftigen konnten. - Der Religion! Und welcher Religion! - Nichts Schlimmeres zu vermeiden, sprangen Tausende in die Fluthen und sind Heilige ! - Geben Sie mir, mein Vater, geben Sie mir diesen Dolch."

Wie kommt dieses Mädchen dazu, nicht etwa äussere Gewalt, sondern Verführung zu fürchten, ihr eigenes warmes Blut, ihre Sinne anzuklagen, „mit den Vorstellungen einer übermächtigen Sinn- 
lichkeit zu ringen?:-1). Schon der Wandsbecker Bote hat dies , eine Ding nicht recht in Kopf bringen können . . . Mich dünkt, ich hätt' an ihrer Stelle nackt durch ein Heer der wollüstigen Teufel gehen. können, und keiner hätte es wagen sollen, mich anzurühren." Gerade gegen diesen Punkt wenden sich denn auch die Vorwürfe neuerer Kritiker zumteil mit besonderer Bitterkeit und Schärfe, Vorwürfe, die einen mehr als einmal an Lessings beherzigenswerte Worte aus dem 73. Stücke der Hamb. Dramaturgie erinnern : „Ich bin überzengt, dass das Auge des Künstlers grösstenteils viel scharfsinniger ist, als das scharfsinnigste seiner Betrachter. Unter zwanzig Einwürfen, die ihm diese machen, wird er sich von neunzehn erinnern, sie während der Arbeit sich selbst gemacht und sie anch schon sich selbst heantwortet zu haben." - Die einen behaupten nach Herders Vorgang, Lessing habe die zarte Weiblichkeit nicht gekannt, andere, er habe sich rücksichtslos gegen die Reinheit der jungfräulichen Natur, gegen die Wahrheit jungfräulicher Empfindung versündigt, sprechen von einem durchaus unmotivierten und eben darum um so hässlicheren und die Teilnahme schwächenden Makel, der auf Emilia falle, von einer erkaltenden und abstossenden Wirkung der nackten Formulierung dieses Seelenzustandes ${ }^{2}$ ). Nur dann ist man so nachsichtig, dieses ,erste Erwachen einer bisher latent gebliebenen glühenden Sinnlichkeit $"$ für erträglich zu halten, wenn man annehmen darf, dass der Prinz, dieses geborenen Verführers dämonische Persönlichkeit verborgene Tiefen der Leidenschaft in Emilia aufgewühlt, sie so in seinen Zauberkreis gebannt hat, dass sie sich ron ihm umstrickt, fasciniert sieht wie der bezauberte Vogel von der Schlange $\left.{ }^{3}\right)$. -

1) Fr. Kern, dentsche Dramen als Schullektüre, S. 10 , wo wegen dieses Seelenzustandes Emiliens unser Stück als zur eingehenderen Klassenlektiure gänzlich ungeeignet bezeichnet wird. Über die pädagogische Behandlung vergl. Wendt, der deutsche Unterricht S. 73 und 116.

$\left.{ }^{2}\right)$ So auch Hettner, Geschichte der dentschen Litteratur im 18. Jahrhundert II ${ }^{3}$ S. 538 und Erieh Sehmidt I1, 1, 214. 5) Bulthaupt S. 26.
Einen Punkt aber, der von so einschneidender Bedentung, von so entscheidender Wichtigkeit ist, dass von ihm die Wahrscheinlickeit des Todesentschlusses Emilias abhängt, den sollte Lessing so wenig hervorgekehrt, so ,latent" gelassen haben, etwa um uns am Schlusse noch das, ,armselige Vergnügen einer Überraschung "1) zuteil werden zu lassen? Gottfried Keller, der sich f ü $\mathrm{r}$ eine Neigung ausspricht, sagt selbst: „Um so mehr hätte Lessing die Sache durchsichtiger behandeln sollen, was sich weder die Alten, noch Shakspeare, noch Schiller hätten entgehen lassen." Und Lessing doch wohl erst recht nicht! Denn es steht geschrieben in der Hamb. Dramaturgie: „Für den Zuschauer muss Alles klar sein. Er ist der Vertrante einer jeden Person; er weiss Alles was vorgaht, Alles was vorgegangen ist... Wir wollen es anf der Bühne sehen, wer die Menschen sind, und können es nur aus ihren Thaten sehen." Die Folgerung muss darum anders fallen als bei Bulthaupt. Nicht: trotzdem Lessing diesen Punkt so wenig herausgearbeitet hat, ist die Annahme einer Liebe gerechtfertigt, sondern: weil er es überhaupt nicht gethan, deshalb haben wir kein Recht, uns auf eine solche Annahme zu stützen. Merkwürdig! Bei Besprechung der Schuld der Jungfrau von Orleans argumentiert Bulthaupt ganz anders. ,Schiller, der so planvoll arbeitete, würde entschieden nichts versäumt haben, diesen Punkt genügend hervorzaheben, wenn er ihm schon während des Schaffens als der entscheidende aufgegangen wäre" (S. 281). Aber welcher Dramatiker arbeitete denn planvoller als Lessing, und wo hătte er dies mehr gethan als in der Emilia Galotti? Es hilft alles nichts. wir müssen ohne diese geheime Neigung auszukommen suchen.

Einen radikalen Versuch macht Bertling ${ }^{2}$ ). Ausgehend von der ,,bisher unbeachtet gebliebenen schriftstellerischen Eigentümlichkeit Lessings, in

1) Hamb. Dram. 48.

$\left.{ }^{2}\right)$ "Die Unwahrbeit in Lessings Sehriften" in d. Jahrb. für Philologie und Pädagogik 1888 , S. 535 ff. - Der Erklärungsversuch findet sich schon bei Arnold a o. O S. 16 . 
guter Absicht die Unwahrheit zu gebranchen," und von der merkwürdigen Gewohnheit des Dichters, gerade die edleren Personen seiner Dramen durch irgend eine gutgemeinte Unwahrheit ihren Edelmut und ihre Selbstlosigkeit an den Tag legen zu lassen, nimmt er an, Emilia denke gar nicht daran, dass sie von dem schändlichen Prinzen sich könnte verführen lassen, sie spreche einfach die $\mathrm{Unw}$ a h rheit, um den Vater zu bewegen, dass er ihr das unerträglich gewordene Leben nehme. Sehr ,einfach" in der That, aber auch sehr banausisch! Wäre das des Rătsels Lösung, dann könnte man Lessings Frage: „Wozu die saure Arbeit der dramatischen Form?:" (H. D. 80) an ihn selbst richten. Denn es wäre gerade die innere Wahrscheinlichkeit der Katastrophe, der Angelpunkt des ganzen Stücks mit auf ein Motiv gestellt, das mit dem $\mathrm{Ch}$ a ra k te r Emilias in gar keiner Beziehung steht; es wäre ein Armutszengnis, das der Dichter sich selbst ausstellte, der nicht zu leisten vermocht hätte, was ex selbst gefordert, in der That ,der Abschluss eines gewöhnlichen Possenspiels":1). Man berufe sich dceh anch nicht auf die Worte am Schlusse der Tragödie :

Emilia: Nicht Sie, mein Vater - Ich selbst - ich selbst -

Odoardo: Nicht Du, meine Tochter — nicht Du! Gehe mit keiner Unwahrheit aus der Welt.

Emilias Worte enthalten mit nichten eine Unwahrheit; es ist ihr mit ihrer Behauptung bitter ernst. Denn sie hat dem zaudernden, zögernden Vater den Dolch in die Hand gedrückt, ihm die That wider seinen Willen entrissen; sie kann darum auch in vollem Ernste diese That die ihrige nennen.

\section{2.}

Wenn alle Fäden des dramatischen Gewebes durch den Kern der Charaktere hindurchgehen, diese die Handlungen und Schicksale der Personen bestimmien müssen, so kann die Lōsung nur auf diesem

1) Jeep ,Der Tod der Emilia Galotti" in d. Jahrb. f. Phil. u. Päd. 1890, S. 580 ff.
Wege gesucht werden. Denn: „Hab ich des Menscher Kern erst untersucht, so weiss ich auch sein Wollen und sein Handeln." Daran hat sich Kuno Fischer gehalten. Mit Recht legt er zur Erklärung des Todesentsehlusses Emilias besondern Nachdruck auf den ehristlichen Charakter der Jungfrau. In dieser drangvoll fürchterlichen Enge erscheint dem frommen Mädchen der freiwillige Tod als der einzige Ausweg, als die einzige Rettung seiner Seele vor dem Verderben auf ewig. Verlassen, wie es ist, von aller und jeder irdischen Hilfe, ruft sein frommer Glaube ihm zu: ,In einer solchen Gefahr giebt es nur eine solche Rettung! Flüchte Deine Seele zu Gott!: Die Behauptung ${ }^{1}$ ), dass w ir nicht einsehen, dass der Heldin in ihrer Lage unbedingt kein anderer Ausweg bleiben soll als der selbstgewählte Tod, verrückt den Standpunkt der Betrachtung. Es muss wiederholt werden: „Die Gesinnungen müssen dem angenommenen Charakter der Person, welche sie äussert, entsprechen; sie kömnen also das Siegel der a bsoluten Wahrheit nicht haben; genug, wenn sie poetiseh wahr sind." Und wie rechtfertigt doch Schiller im 12. Briefe über Don Carlos den Entschluss seines Posa?: „Es kommt hier nicht darauf an, wie not hw endig, wie natürlich, wie nützlich diese Auskunft in der That war, sondern wie sie demjenigen vorkam, der sie zu ergreifen hatte. Es ist also weit weniger die Lage der Dinge, als die Gemūthsverfassung dessen. auf den diese Dinge wirken, was hier in Besacht kommen muss. Sind die Ideen, welche den Marquis zu diesem Heldenentschluss führen, ihm geläufig und bieten sie sich ihm leicht und mit Lebhaftigkeit dar, so ist der Entschluss anch weder gesucht noch gezwungen".

Wie steht es nun aber mit dem heikelsten Punkte des Dramas, auf den schon so viele sittliche und ăsthetische Entrüstung verschwendet wurde, mit Emilias Furcht vor Verführung? Kuno Fischer leitet diese aus den ungewöhnlichen und ersten Eindrïcken der grossen und glänzenden Welt im

1) Franz, der Autbau der Handlung in den klassischen Dramen S. 41 . 
Hause der Grimaldi her, die auf die kindliche, harmlose und phantasievolle Natur ihre Macht ausgeübt und wie ein lockender Zauber fortgewirkt haben, einer Welt, deren innerste Verdorbenheit sie völlig erkannt hat, so dass sie nach der ersten Erfahrung, die sie an sich selbst gemacht, sich jetzt nicht für gerüstet und fähig hält, eine zweite Probe zu bestehen ${ }^{1}$ ). .Aus Furcht vor ihrem den ersten Eindrücken erliegenden Temperament begehrt Emilia - der der Dichter einen Reiz der Sinnlichkeit ins Blut gelegt - den Tod *, sagt Erich Schmidt ${ }^{2}$ ). Allein mit solchen Reflexionen würde Emilia eine Selbst-, Welt- und Menschenkenntnis, eine Einsicht in ihre eigene und die menschliche Natur bekunden, wie sie das trotz jenes Erlebnisses doch noch zu unerfahrene Mädchen schlechterdings nicht haben kann. Diese Emilia denkt viel zu philosophisch, und ,die jungfräulichen Philosophinnen sind gar nicht nach meinem Geschmack. Ich kenne an einem unverheiratheten Mädchen keine höheren Tugenden als Frömmigkeit und Gehorsam, antwortet Lessing seinem Bruder Karl, der nach der Lektüre der noch unvollendeten Tragödie es rationalistisch getadelt hatte, dass er seine Emilia nur als fromm und gehorsam schilderte, dem ihre Frömmigkeit und ,das Pedantische der Religion" etwas verảchtlich vorkam ${ }^{3}$ ). Anders des Bruders Urteil über die vollende te Tragödie $e^{4}$ : „Du erinnerst Dich noch, dass mir die Emilia im Anfang nicht so vorzüglich gefallen. Da hast mir daher einige Deiner Gründe angeführt, von denen aber keiner Stich zu halten schien, als der letzte, da Da sagtest: „,,Am Ende wird denn anch freilich der Charakter der Emilia interessanter, und sie selbst thätiger." ". Denn das ist nicht allein geschehen, sondern ... der Schluss hätte auch nicht so werden können, wenn $\mathrm{Du}$ sie nicht von Anfang an so geschildert hättest. Höchst religiös, die Tugend der Keusehheit für die hőehste

1) „Ist sie doch Italienerin" findet Jeep für gut erläuternd hinzuzufugen.

2) $11,1 \mathrm{~S}, 214$ u. 203.

3) 1. Febraar 1772 , Hempel XX, S. 553 n. 551 .

4) 12. März 1772, Hempel XX, 2 S. 570 u. 571.
Tugend haltend ist Emilia; un 1 das letzte hat sie bloss durch ihre fast blinde Anhänglichkeit an die katholische Religion werden können." Hier liegt für uns die Entscheidung. Auch ihre Furcht vor Verführung erscheint uns als ein Ausfluss ihres frommen Glaubens. Das ist nicht eine aus dem tiefsten Grunde einer sinnlich angelegten Natur organisch herauswachsende, durch Erfahrung und Selbsterkenntnis gezeitigte Furcht, nein, die fromme Jungfrau fürchtet, weil ihre Religion sie die Verführbarkeit der menschlichen Natur fürchten lehrte ${ }^{1}$ ). Diese lehrte sie: „Der Geist ist w i $11 \mathrm{ig}$ " -: ,Will mich reissen, will mich bringen : will! will! als ob wir keinen Willen hatten, mein Vater!... Gewalt! Gewalt! wer kann der Gewalt nicht trotzen ?* Aber sie lehrte sie auch: "Das $\mathrm{Fle}$ is $\mathrm{ch}$ ist $\mathrm{se} \mathrm{hw} \mathrm{a} \mathrm{ch}$. und ,das Fleisch gelüstet wider den Geist", und d a r um sagt sie : "Ich habe Blut, mein Vater, so juggendliches, so warmes Blut, als eine. Auch meine Sinne sind Sinne. Ich stehe für nichts. Ich bin für nichts gut." Die Gelegenheit, bei der diese Befürchtung sich verwirklichen könnte, sieht sie schandernd vor sich: sie soll in das Haus der Grimaldi. Schon einmal hat sie hier die Freuden der Welt erblickt, ein Erlebnis, das sie in ihres Herzens Fröm igkeit als ein sündhaftes empfand und das dar $\mathrm{um}$ einen Tumult in ihrer Seele hervorrief, den nur ,die strengsten Übungen der Religion besänftigen konnten“. „Der Religion! Und weleherReligion?:* Der-

1) Vergl. die wenn auch nicht durchaus vergleithbare Scene aus Gosthes Götz von Berlichingen (I). Maria: Ich bitt Euch, lasst mich. Einen Kizs hab ich Euch zum Gottespfennig erlaubt. Ihr scheint aber schon von dem Besits nehmen zu wollen, was nur unter Bedingungen Ener ist. Weislingen: Ihr seid za streng, Maria! Unschuldige Liebe erfrent die Gottheit, statt sie zu beleidigen. Ma ria: Es sei! Aber ich bin nicht dadurch erbant. Man lehrte mich, Liebkosungen seien wie Ketten, stark durch ihre Verwandtschaft, und Mädchen, wenn sie liebten, seien schwächer als Simson nach dem Verlust seiner Locken. Weislingen: Wer lehrte Euch das? Maria: Die Äbtissin meines Klosters... 
jenigen, die sie nicht bloss die Schwachheit des Fleisches fürchten lehrte und ihr in einer Lage, wo noch viel mehr als am Morgen ,fremdes Laster sie wider ihren Willen zur Mitschuldigen macht", den Tod, der Sünde Sold, als religiöse Pflichterfüllung erseheinen lässt, sondern die ihr auch für eine solche That den herrlichsten Lohn, die Märtyrerkrone, verheisst. „Nichts Schlimmeres zu vermeiden, sprangen Tausende in die Fluthen und sind Heilige!" Dem gegenüber ist dieses Leben für sie eine völlig wertlose Ware, die sie, die wie Philotas ,zu sterben weiss", von sich wirft. „Dieses Leben ist alles, was die Lasterhaften haben. Mir, mein Vater, mir geben Sie diesen Dolch." Es geht ein asketischer Zug durch Emilias Worte, und es ist vielleicht auch kein „Zufall", dass sie bei den Dominikanern in die Kirche ging.

Somit beruht Emilias ganzes Denken und Handeln auf jenen zwei Charakterzügen, die Lessing mit Beziehung auf unser Drama als die höchsten Tugenden eines Mädchens bezeichnete. Der Gehorsam erklärt ihre tragische "Schuld", die F r ö m i g ke it ihre Furcht vor Verführung und ihren Todesentschluss.

\section{3.}

Auch in der That 0 doardos hat man die innere Wahrscheinlichkeit vermisst. Und doch ist auch sein Charakter gleich von vornherein gerade auf diese That angelegt. Der Mann, der uns bei seinem ersten Auftreten den Ort bezeichnet, wo er , ,am tödtlichsten zu verwunden ist", den der blosse Gedanke schon in Wuth setzt, der schon so wild war, als er hörte, dass der Prinz seine Tochter jüngst ,nicht ohne Missfallen" betrachtete, der in seinem Zorn den unschuldigen Gegenstand des Verbrechens mit dem Verbrecher verwechselt haben würde, der, durch all' das Erlebte und Empfundene, durch all' die furchtbaren Seelenkämpfe, dureh Orsinas moralische Dolchstosse, durch Marinellis und des Prinzen schurkisehe Anschläge an die Grenze des Wahnsinns gebracht, das Schicksal seiner an sich selbst verzweifelnden Tochter in dem Bilde einer zerpflückten Rose vor sich sieht und schliesslich mit Hinweis auf des alten Römers That den furchtbaren Vorwurf eines pflichtvergessenen Vaters sich -machen hört: der Mann soll ,bei diesem Grade der Leidenschaft, bei dieser Lage der Sachen nicht von einem so fatalen Strom dahingerissen werden können, Dinge zu begehen, die wir bei kaltem Geblüte noch so weit von uns entfernt $z u$ sein glauben"?") Und nun kommt man mit der verwünscht gescheiten Frage, ob es denn, in Guastalla nicht auch noch ordentliche Menschen giebt, die das Hofleben noch nicht corrumpiert hat, unter deren Obhut Emilia hätte bleiben können"2). Gewiss giebt es die; die Dominikaner z. B. wären zweifellos die vortrefflichsten Tugendwächter gewesen. Auf diesen die verengte Situation mit eins durchbrechenden Gedanken wäre Odoardo, vielleicht mit einiger Beihilfe Claudias, sicherlich auch noch gekommen, hätte er nur nicht so unüberlegt zugestossen und noch ein Weilchen zugewartet. Ja, wenn dieses Warten diesen Odoardos nur nicht gar so schwer, so ganz unmöglich wäre! Wem das nicht einleuehtet, der lasse sich durch die Ausführungen belehren, mit denen Schiller seinen Posa verteidigt: „Was hätte der ruhige Zusehauer nicht gekonnt, und wie viel weiser und klüger würde dieser .... gewirthschaftet haben! Schade nur, dass sich der Marquis weder dieser glücklichen Kaltblütigkeit, noch der Musse zu erfreuen hatte, die zu einer so vernünftigen Berechnung nothwendig war" - oder er beherzige Kuno Fischers klassische, einem Lessing kongeniale Worte $\left.{ }^{3}\right):$, Wenn in derKette des tragischen Causalnexus überall das Ungefähr ausgeschlossen sein und alles so geschehen soll, dass es nicht anders geschehen

\section{1) Hamb. Dram. 82.}

2) Bulthrupt 824.

3) S. 256 bis 258 . Ähnlieh Nietzsche ,Menschliches, Allzumenschliches" I S, 63 . Nr. 61 „,Warten können!" “ „Das Warten-können ist so schwer, dass die grössten Dichter es nicht verschmaiht haben, das Nicht-warten-können zum Motiv ihrer Diehtungen zu machen ... . Die Leidenschaft will nicht warten" .... 
konnte: so muss auch jede tragische Handlung ihren genau bestimmten $\mathrm{Z}$ eit pun $\mathrm{kt}$ haben. Was nicht jetzt geschieht, unterbleibt für immer; der einmal verlorene Moment ist unwiederbringlich verloren. Was geschieht, geschieht jetzt oder nie! Jeder Moment ist gekettet an die vorhergehenden und bindet die folgenden. Es gibt in der Tragödie keine Opportunităt, keine so bedăchtige Wahl und günstige Lage der Zeitpunkte, dass alle schlimmen Folgen der Handlung weislich verhütet werden: keine Zeitpunkte, die man erwartet, wie die bequemen Spaziergänger das gute Wetter. Die tragischen Leidenschaften gehen nicht spazieren, sie sind eilig und haben keine Zeit zu verlieren, wie der Prinz das Todesurteil ungelesen unterschreiben will, um schneller in Emiliens Nähe zu sein. Die Zeit in der Tragödie ist furchtbar, wie das Schicksal selbst, und ich kenne kein Trauerspiel, worin mir diese Furehtbarkeit so eingeleuchtet hätte, wie hier, keines, worin jede Handlung und jede Unterlassung so wie hier an ihren Zeitpunkt gekettet wäre. Dies gilt auch von dem Moment, worin Emilia den Entschluss zu sterben fasst; auch von dem Augenblick, worin Odoardo sie tödtet. Dadurch wird die Nothwendigkeit der Handlungen nicht abgemindert, sondern in Wahrheit erst vollendet."

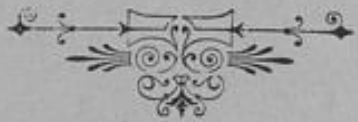


konnte: so muss auch jede tragisehe Handlung ihren genau bestimmten $\mathrm{Z}$ eitpu $\mathrm{nkt}$ haben. Was nicht jetzt geschieht, unterbleibt für immer; der einmal verlorene Moment ist unwiederbringlich verloren. Was geschieht, geschieht jetzt oder nie! Jeder Moment ist gekettet an die vorhergehenden und bindet die folgenden. Es gibt in der Tragödie keine Opportunităt, keine so bedächtige Wahl und günstige Lage der Zeitpunkte, dass alle schlimmen Folgen der Handlung weislich verhütet werden: keine Zeitpunkte, die man erwartet, wie die bequemen Spaziergänger das gute Wetter. Die tragischen Leidenschaften gehen nicht spazieren, sie
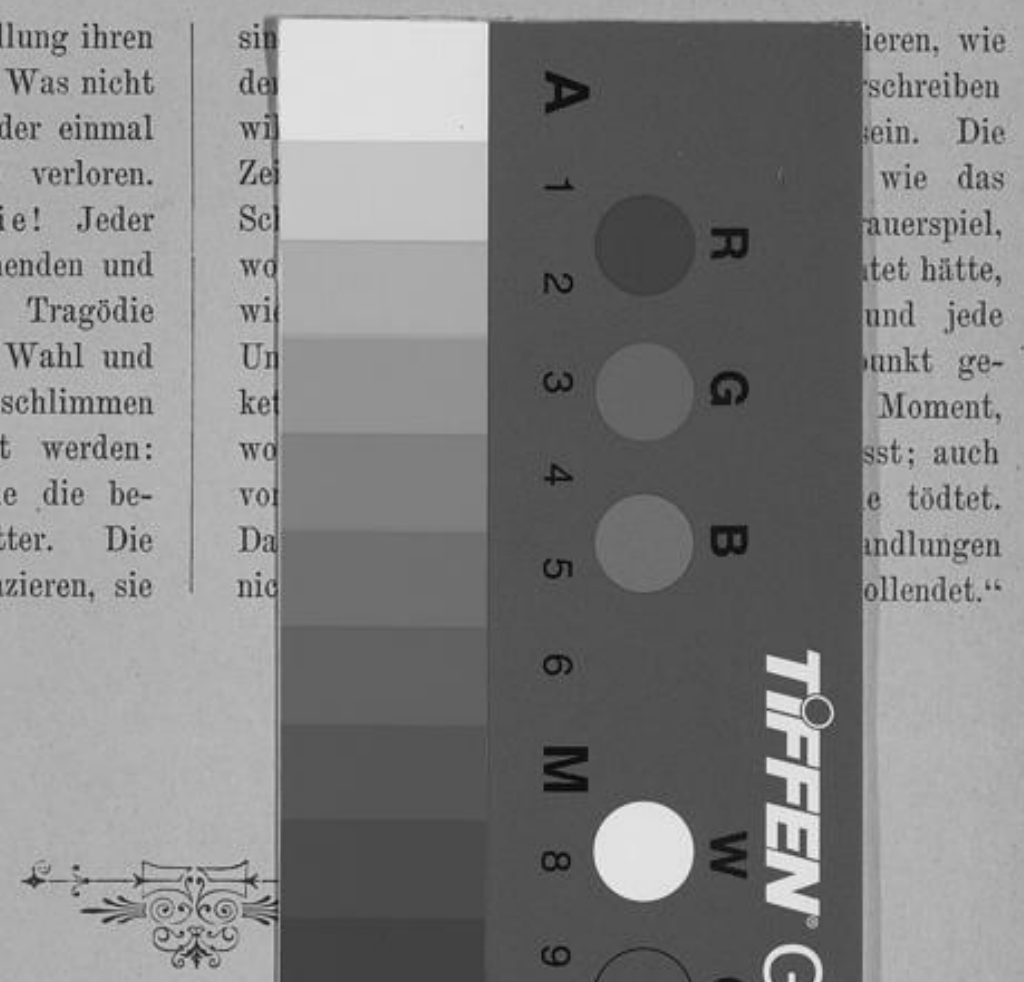

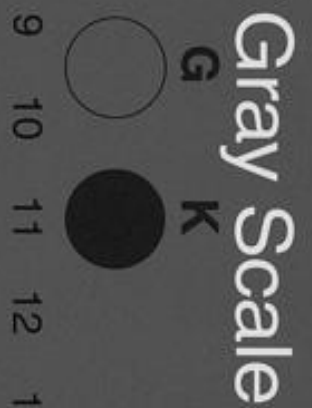

$\vec{\omega}$
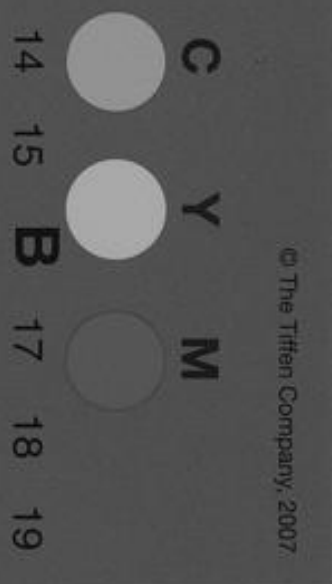


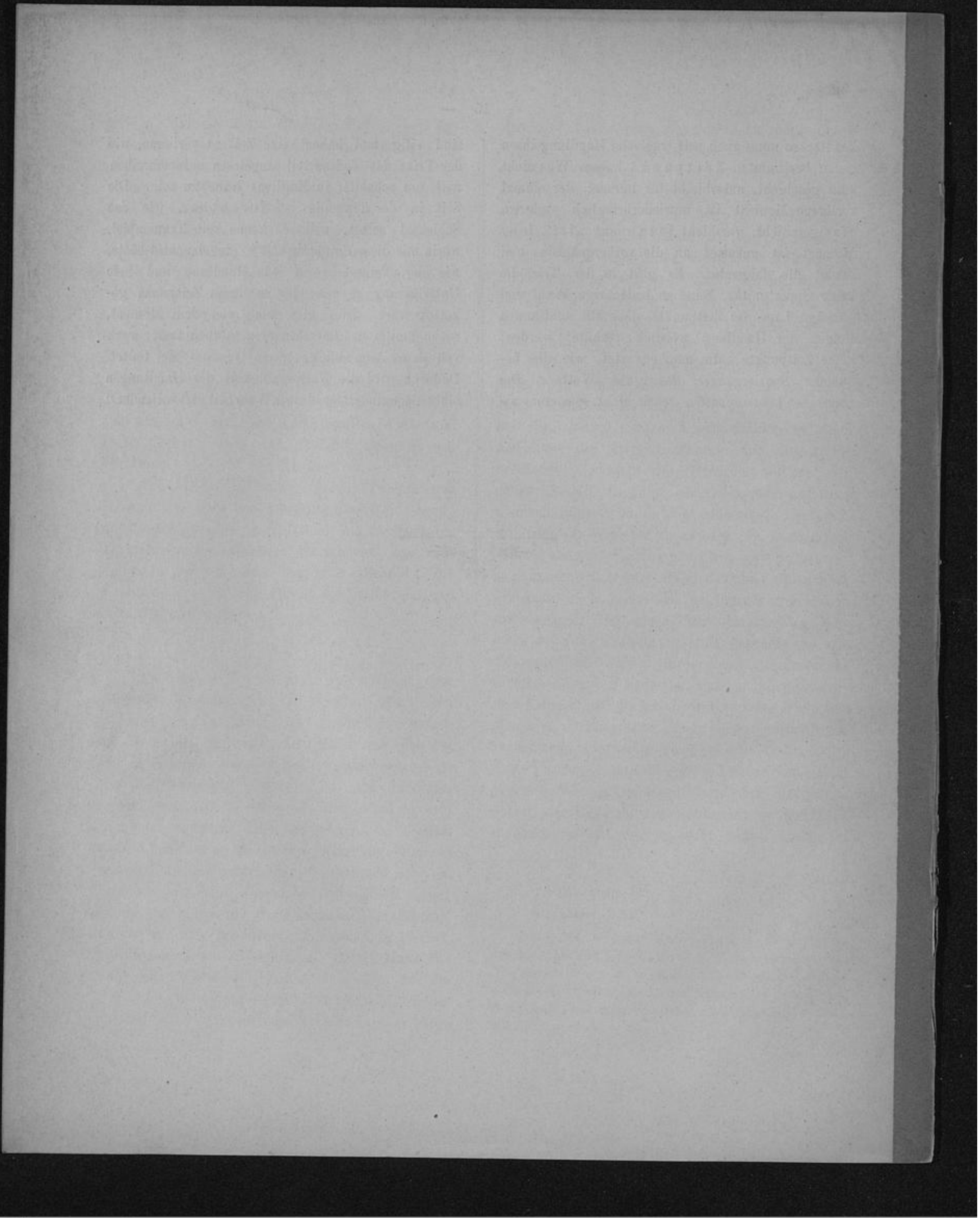

